\title{
Planimetry of aortic valve area using multiplane transoesophageal echocardiography is not a reliable method for assessing severity of aortic stenosis
}

Yvette Bernard, Nicolas Meneveau, Alain Vuillemenot, Dominique Magnin, Thierry Anguenot, François Schiele, Jean-Pierre Bassand

\begin{abstract}
Objective-To assess the reliability of aortic valve area planimetry by multiplane transoesophageal echocardiography (TOE) in aortic stenosis.

Design-Study of the diagnostic value of aortic valve area planimetry using multiplane TOE, compared with catheterisation and the continuity equation, both being considered as criterion standards. Setting-University hospital.
\end{abstract}

Patients-49 consecutive patients (29 male, 20 female, aged 44 to 82 years, average 66.6 (SD 8.5)), referred for haemodynamic evaluation of an aortic stenosis, were enrolled in a prospective study. From this sample, 37 patients were eligible for the final analysis.

Methods-Transthoracic and multiplane transoesophageal echocardiograms were performed within 24 hours before catheterisation. At transthoracic echo, aortic valve area was calculated by the continuity equation. At TOE, the image of the aortic valve opening was obtained with a $30-65^{\circ}$ rotation of the transducer. Numerical dynamic images were stored on optical discs for off-line analysis and were reviewed by two blinded observers. Catheterisation was performed in all cases and aortic valve area was calculated by the Gorlin formula.

Results-Feasibility of the method was $92 \%(48 / 52)$. The agreement between aortic valve area measured at TOE (mean 0.88 (SD 0.35$) \mathrm{cm}^{2}$ ) and at catheterisation $\left(0.79(0.24) \mathrm{cm}^{2}\right)$ was very poor. The same discrepancies were found between TOE and the continuity equation $(0.72(0.26)$ $\left.\mathrm{cm}^{2}\right)$. TOE planimetry overestimated aortic valve area determined by the two other methods. Predictive positive and negative values of planimetry to detect aortic valve area $<0.75 \mathrm{~cm}^{2}$ were $62 \%(10 / 16)$ and $43 \%$ (9/21) respectively.

Conclusions-Planimetry of aortic valve area by TOE is difficult and less accurate than the continuity equation for assessing the severity of aortic stenosis.

(Heart 1997;78:68-73)

Keywords: aortic valve area planimetry; aortic stenosis; multiplane transoesophageal echocardiography
The degree of severity of aortic stenosis is commonly estimated non-invasively by Doppler echocardiography. Transaortic gradients are the most frequently used in clinical practice, especially mean gradient. ${ }^{1-5}$ In patients with severe aortic stenosis and decreased left ventricular function, gradients are low and it is necessary to calculate aortic valve area, either non-invasively by the continuity equation ${ }^{6-8}$ or invasively by the Gorlin formula at catheterisation. ${ }^{9}$ The reliability of the continuity equation has been well demonstrated, but sometimes-especially in elderly patients with poor echogenicity-left ventricular outflow tract diameter is difficult to measure precisely. On the other hand, catheterisation with measurement of simultaneous left ventricle to aorta gradients may be hazardous to such patients in poor clinical condition. It would therefore be useful to have another non-invasive method of determining aortic valve area at our disposal for these difficult cases.

A few years ago, attempts were made to determine aortic valve area by planimetry of the aortic valve opening using monoplane transoesophageal echocardiography (TOE)..$^{1011}$ Aortic valve area measurement was not possible in all patients with this technique. More recently, multiplane TOE has brought new possibilities of aortic valve visualisation, by rotating the imaging plane to the exact position where the aortic valve opening is best seen. ${ }^{12} 13$

The aim of our study was therefore to determine whether aortic valve area planimetry using multiplane TOE was reliable in adults suffering from aortic stenosis.

\section{Methods}

STUDY POPULATION

Forty nine consecutive adult patients referred to our institution for haemodynamic evaluation of calcific aortic stenosis were enrolled in a prospective study. Average (SD) age was $66.6(8.5)$ years (range 44 to 82 ). There were 29 males and 20 females. Most patients were symptomatic, with cardiac failure or dyspnoea on exertion in 28 , chest pain in seven, and syncope in four. Forty four patients were in sinus rhythm and five in atrial fibrillation. Thirteen patients had grade 1+ angiographic aortic regurgitation according to Sellers' classification ${ }^{14}$; patients with aortic 
regurgitation greater than $1+$ were excluded. Six patients had associated coronary lesions, and eight had impaired left ventricular function with an ejection fraction $<0.45$ at angiography. Informed consent was obtained from all the patients.

\section{ECHOCARDIOGRAPHIC EXAMINATION}

All patients were submitted to a transthoracic and a multiplane transoesophageal echocardiographic examination within 24 hours of catheterisation. All echo examinations were performed by three experienced echocardiographers with a Diasonics-Vingmed CFM 800 (Vingmed Sound, Norway).

\section{Transthoracic echocardiography}

Maximum and mean transaortic gradients were recorded from the apical, right parasternal, and suprasternal approaches. A 2.5 to $3.25 \mathrm{MHz}$ multifrequency imaging transducer with orientable continuous wave Doppler was first used, with the help of colour guided continuous wave Doppler; this allowed alignment of the beam on the aortic jet, visualised by the maximum of aliasing and turbulences coded in green. ${ }^{15}$ A $2 \mathrm{MHz}$ Pedof transducer (Vingmed Sound, Norway) was then used if necessary. Left ventricular outflow tract diameter was measured by bidimensional echo imaging in the parasternal long axis view and its flow was recorded in the five chamber apical view by pulsed Doppler. The simplified Bernouilli equation was used to estimate gradients from transaortic velocity curves. Aortic valve area was calculated by the continuity equation using the time-velocity integral of aortic valve and left ventricular outflow tract spectral curves. ${ }^{6-8}$

\section{Multiplane TOE}

Multiplane TOE using a $5 \mathrm{MHz}$ annular phased array probe was performed immediately after transthoracic examination. Patients received $10 \mathrm{mg}$ of diazepam orally one hour before TOE, and then local anaesthesia. After the standard monoplane examination at mid-oesophagus level, the transducer was alternately advanced and withdrawn, and retroflexed and anteflexed; the imaging plane was rotated from $0^{\circ}$ to $180^{\circ}$ until the best image of the aortic valve opening in the short axis view was obtained. This was generally achieved with a $30^{\circ}$ to $65^{\circ}$ rotation of the plane $\left(0^{\circ}\right.$ to $30^{\circ}$ in seven patients). A thorough examination of the smallest orifice at the time of maximum opening in early systole was performed, requiring a very precise rotation of the imaging plane and a correct level position. Gain settings were adjusted as low as possible without losing definition of the commissural edges. The aortic valve image was magnified using the zoom mode. In

Table 1 Values of aortic valve area determined by the different methods

\begin{tabular}{llll}
\hline Variable & No of patients & Mean $(S D)\left(\mathrm{cm}^{2}\right)$ & Range $\left(\mathrm{cm}^{2}\right)$ \\
\hline TOE planimetry & 37 & $0.88(0.35)$ & 0.30 to 1.66 \\
Continuity equation & 37 & $0.70(0.26)$ & 0.33 to 1.52 \\
CE TOE & 22 & $0.70(0.24)$ & 0.38 to 1.37 \\
Catheterisation & 37 & $0.79(0.24)$ & 0.43 to 1.48 \\
\hline
\end{tabular}

CE TOE, aortic valve area measured by continuity equation using left ventricular outflow tract diameter measured at transoesophageal echocardiography.
22 patients, the left ventricular outflow tract diameter was measured when a perfect view of the outflow tract was obtained, that is, with an imaging plane rotation from $90^{\circ}$ to $140^{\circ}$. Aortic valve area was then recalculated using the continuity equation with this diameter. No incidents occurred during the procedure.

Images were recorded on VHS videotapes and dynamic numerical images were stored on optical discs with a minimum of two cineloops for each patient. Cineloops were recorded when an optimal image of aortic valve opening, that is, the minimum orifice area, was obtained. Images were reviewed at least three months after the initial examination by a blinded experienced echocardiographer, who was unaware of any information or results concerning the patients. The average of a minimum of five measurements per patient were used for correlations.

\section{CATHETERISATION}

Right and left heart catheterisation was performed in all patients. Simultaneous left ventricle to aorta maximum and mean gradients were obtained in all but two cases, either by crossing the aortic valve through the retrograde femoral approach with a double lumen $8 \mathrm{~F}$ Cordis catheter, or in a few cases by transseptal catheterisation, using two fluid filled catheters, one placed in the left ventricle and the other in the aorta. Gradients were always measured at the beginning of catheterisation, before any contrast medium injection. Cardiac output was determined by thermodilution, averaging five measurements. Aortic valve area was determined by the Gorlin formula. ${ }^{9}$ All pressure tracings and valve areas were processed with an IBM AT 386 computer connected to a Quinton-Q-Cath (Quinton Instrument Co, Seattle, USA). All calculations were subsequently controlled, especially ejection time measurement, and all haemodynamic aortic valve areas were recalculated with verified values.

\section{ANALYSIS OF RESULTS}

The results obtained at blinded analysis of TOE images were compared to the haemodynamic aortic valve area and to the continuity equation. Aortic valve area determined by the continuity equation was also recalculated with the left ventricular outflow tract diameter measured at TOE (CE TOE) in 22 patients and the results were compared to transoesophageal aortic planimetry, haemodynamic data, and the usual continuity equation data.

To determine intraobserver variability, 12 TOE were randomly selected and reanalysed at least three months later by the same echocardiographer. To determine interobserver variability, 11 patients were randomly selected and their TOE were independently analysed by two blinded observers.

\section{STATISTICAL ANALYSIS}

The agreement between the three methods of aortic valve area measurement was evaluated by the Bland and Altman method ${ }^{16}$ : the difference between two methods was plotted against 
Table 2 Agreement between aortic valve area measured by the different methods of echocardiography and by catheterisation

\begin{tabular}{lllll}
\hline & & \multicolumn{3}{l}{ Limits of agreement } \\
\cline { 3 - 4 } Variables & $M D\left(\mathrm{~cm}^{2}\right)$ & $S D\left(\mathrm{~cm}^{2}\right)$ & $M D-2 S D(95 \% \mathrm{CI})$ & $M D+2 S D(95 \% C I)$ \\
\hline Cath/TOE & -0.09 & 0.32 & $-0.73(-0.91 ;-0.55)$ & $+0.55(+0.37 ;+0.70)$ \\
CE/TOE & -0.16 & 0.30 & $-0.76(-0.94 ;-0.58)$ & $+0.44(+0.26 ;+0.62)$ \\
Cath/CE & +0.07 & 0.18 & $-0.27(-0.37 ;-0.17)$ & $+0.43(+0.33 ;+0.53)$ \\
Cath/CE TOE & +0.08 & 0.18 & $-0.28(-0.38 ;-0.18)$ & $+0.44(+0.36 ;+0.54)$ \\
CE/CE TOE & +0.02 & 0.11 & $-0.22(-0.28 ;-0.16)$ & $+0.24(+0.18 ;+0.30)$ \\
Meas 1/Meas 2 & -0.07 & 0.21 & $-0.49(-0.57 ;-0.41)$ & $+0.35(+0.27 ;+0.43)$ \\
Oper 1/Oper 2 & -0.13 & 0.21 & $-0.55(-0.57 ;-0.41)$ & $+0.35(+0.27 ;+0.43)$ \\
\hline
\end{tabular}

Cath, catheterisation; CE, continuity equation; $C B$ TOE, continuity equation with left ventricular outflow tract diameter measured at transoesophageal echocardiography; CI, confidence interval; Meas, measurement (Meas 1/Meas 2, repeatability of TOB planimetry); Oper, operator (Oper 1/Oper 2 , interobserver variability).
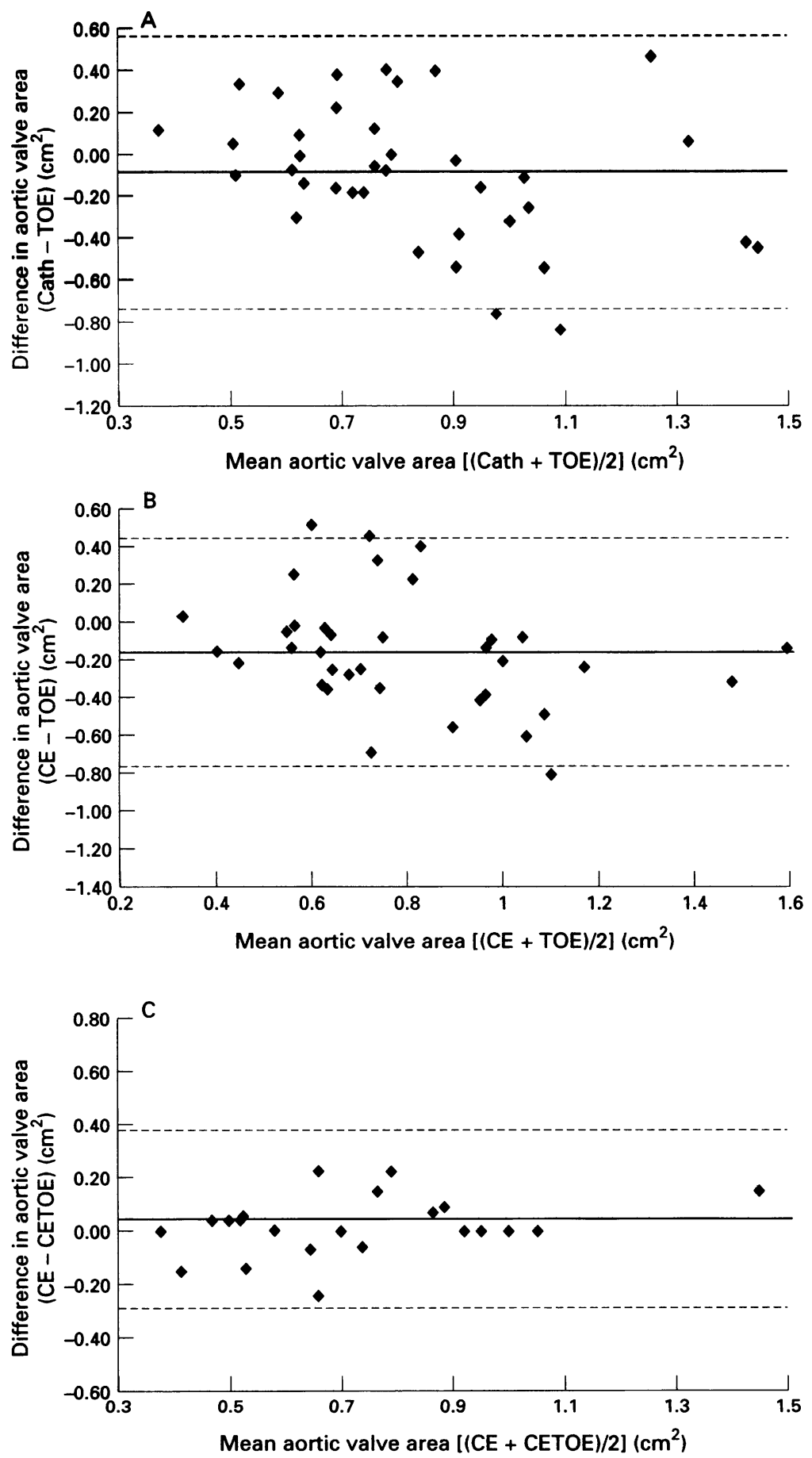

Figure 1 Agreement between aortic valve area measured by the different methods. (A) TOE $v$ catheterisation agreement. (B) TOE $v$ continuity equation. (C) CE TOE $v$ continuity equation. For each graph, the difference between the two comparative measurements is plotted against their mean, according to Bland and Altman. Dashed lines represent limits of agreement (mean difference $\pm 2 S D$ ). TOE, transoesophageal echocardiography; CE, continuity equation. their mean, as the mean of two measurements is the best estimate of the true value; the degree of agreement was assessed by calculating the bias, estimated by the mean difference, and the standard deviation of the differences. If differences within the limits of agreement (that is, mean difference \pm 2 SD) are not clinically relevant, the two methods are interchangeable. The precision of estimated limits of agreement was evaluated using $95 \%$ confidence intervals, assuming that the differences follow an approximately normal distribution.

Repeatability of TOE measurements (that is, variation in repeated measurements on the same subject) was also assessed using the statistical method of Bland and Altman. We would expect $95 \%$ of pairs of measurement to lie closer together than 2 SD above or below the mean difference; hence this was regarded as a good index of the degree of repeatability of data from the two aortic valve area methods of measurement. ${ }^{17}$

A receiver operating characteristic (ROC) curve was drawn to determine threshold planimetered aortic valve area which corresponds to the $0.75 \mathrm{~cm}^{2}$ adopted as a criterion of severity using continuity equation. Sensitivity and specificity of the method were then calculated from this threshold value.

\section{Results}

Five patients were excluded because of atrial fibrillation, which decreases the correlation between Doppler derived and haemodynamic gradients. ${ }^{18}$ In two others, crossing of the aortic valve was not achieved and their clinical condition was too poor to submit them to transseptal catheterisation. In one patient, the continuity equation was not obtained due to a lack of echogenicity. Aortic valve area planimetry was judged impracticable in four patients with very calcified valves. All in all, 12 patients were excluded.

The feasibility of TOE planimetry was $92 \%$ (48/52), while the feasibility of the continuity equation was $98 \%(51 / 52)$.

Aortic valve area measurements determined by the different methods are reported in table 1 .

\section{AGREEMENT BETWEEN AORTIC VALVE AREA} VALUES

The agreement between aortic valve area values measured by echocardiography (TOE planimetry, continuity equation, CE TOE) and catheterisation is shown in fig 1 . Table 2 


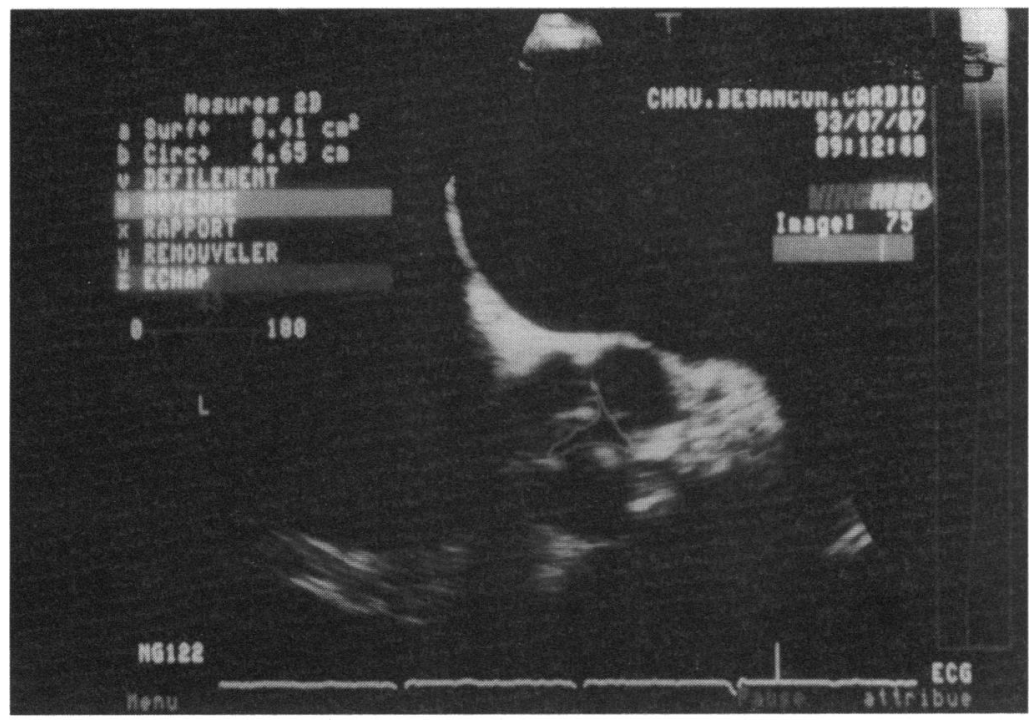

Figure 2 Example of a calcific tricuspid aortic valve (multiplane TOE, rotation angle $\left.43^{\circ}\right)$.

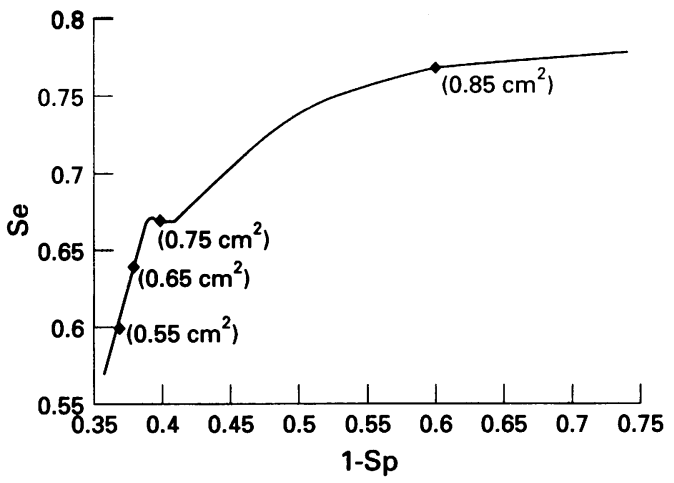

Figure 3 Receiver operating characteristic (ROC) curve analysis of the accuracy of transoesophageal echocardiography (TOE) in determining the severity of aortic stenosis, according to the $0.75 \mathrm{~cm}^{2}$ criterion of severity considered using the continuity equation. The values between brackets represent the respective threshold values of TOE aortic valve area. The area under the curve is proportional to the usefulness of TOE in discriminating correctly between severe and non-severe aortic stenosis, the area being $1 \cdot 0$ with the ideal test.

summarises these results in terms of mean difference $(M D)$ and \pm 2 SD. Mean differences were $-0.09,-0.16$, and $+0.08 \mathrm{~cm}^{2}$ for TOE $v$ catheterisation, TOE $v$ continuity, equation, and CE TOE $v$ catheterisation, respectively.

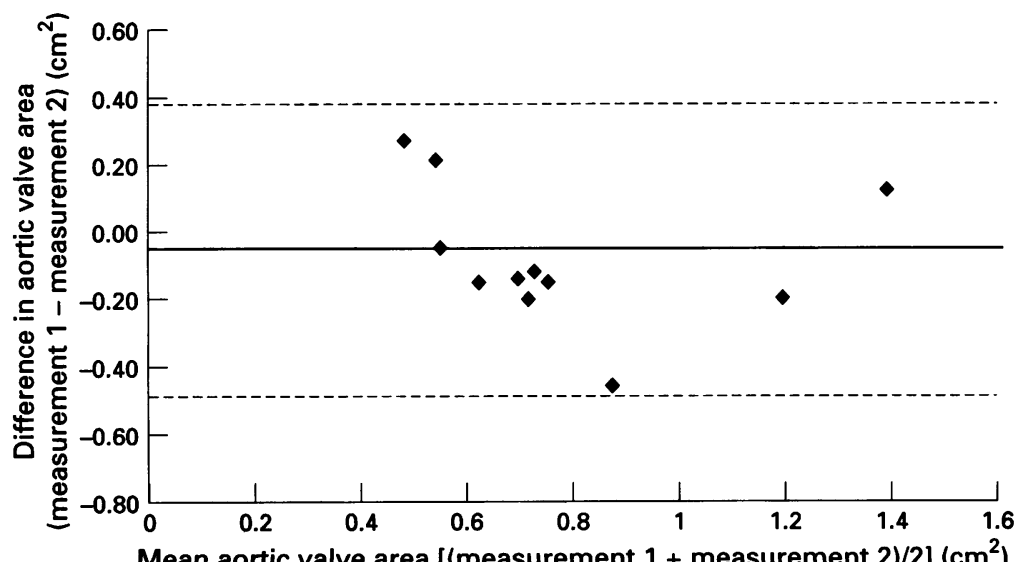

Figure 4 Repeated measurements of aortic valve area on the same subject using transoesophageal echocardiographic planimetry.
The limits of agreement (that is, $\mathrm{MD}-2 \mathrm{SD}$ to $\mathrm{MD}+2 \mathrm{SD}$ ) were -0.73 to $+0.55 \mathrm{~cm}^{2}$, -0.76 to $+0.44 \mathrm{~cm}^{2}$, and -0.28 to $+0.44 \mathrm{~cm}^{2}$ for TOE $v$ catheterisation, TOE $v$ continuity equation and CE TOE $v$ catheterisation, respectively. The precision of limits of agreement determined, using $95 \%$ confidence intervals, showed a wide range of values, reflecting the small sample size and the great variation of the differences for TOE $v$ catheterisation and TOE $v$ continuity equation. Discrepancies were also found for continuity equation $v$ catheterisation and for CE TOE $v$ catheterisation, but to a lesser extent. Regarding data compared in TOE $v$ catheterisation, the $95 \%$ confidence intervals were -0.91 to $-0.55 \mathrm{~cm}^{2}$ and +0.37 to $+0.73 \mathrm{~cm}^{2}$ for the lower and upper limits of agreement, respectively. Thus even with the most optimistic interpretation there can be considerable discrepancies between TOE and catheterisation measurements $(-0.55$ to +0.37$)$, as well as between TOE and the continuity equation, and the lack of agreement between these methods is unacceptable in clinical practice. TOE planimetry is likely to overestimate aortic valve area obtained by catheterisation. The comparison between the continuity equation and the Gorlin formula, as well as between CE TOE and the Gorlin formula, shows better but still unsatisfactory results, with relatively wide $95 \%$ confidence intervals, and with an underestimation of aortic valve area determined at catheterisation. Conversely, aortic valve area determined by the usual continuity equation and by CE TOE correlated very well, which reflects the good agreement between values for left ventricular outflow tract diameter measured by transthoracic and transoesophageal echocardiography.

The agreement between methods did not depend on the characteristics of aortic valve. Thus it was not better in tricuspid valves (26 patients) (fig 2) than in bicuspid valves (11 patients), and vice versa. In the same way, it was not possible to identify a subgroup of patients with less calcified valves and a better correlation; however, almost all the patients had significant calcifications.

Threshold planimetered area corresponding to the $0.75 \mathrm{~cm}^{2}$ criterion of severity using the continuity equation was determined by means of an ROC curve. The ROC curve (fig 3) shows the variation in TOE sensitivity and specificity according to the threshold value considered. The highest sensitivity simultaneously combined with the highest specificity approximately corresponds to the threshold value of $0.75 \mathrm{~cm}^{2}$.

The sensitivity and specificity of TOE planimetry to predict severe aortic stenosis, that is aortic valve area $<0.75 \mathrm{~cm}^{2}$, did not exceed $45 \%(10 / 22)$ and $60 \%(9 / 15)$ respectively. Predictive positive and negative values were $62 \%(10 / 16)$ and $43 \%(9 / 21)$.

Repeatability determined as variations in two repeated measurements on the same subject was assessed on a series of 12 patients. Figure 4 shows difference plotted against the mean for each subject, according to the Bland and 
Altman method. The mean difference did not differ significantly from zero, and all the differences were less than 2 SD, which meets the definition of good repeatability.

Interobserver variability, assessed using the same method, clearly demonstrated a weak correlation between the two operators' measurements. These discrepancies confirm the poor interobserver reproducibility of TOE planimetry.

\section{Discussion}

Attempts to measure aortic valve area by planimetry in the short axis view have been made by the transthoracic approach, either by means of bidimensional echo, ${ }^{19}$ or pulsed Doppler, ${ }^{20}$ or colour Doppler cartography. ${ }^{21}$ These methods are seldom usable in clinical practice. When TOE appeared, some investigators measured aortic valve area with monoplane TOE. ${ }^{10} 11$ However, visualisation of the aortic valve in the short axis is not always obtained with monoplane or biplane views. Recently, multiplane TOE has brought new insights to aortic valve visualisation: with this device, the rotation of the imaging plane degree by degree from $0^{\circ}$ to $180^{\circ}$ allows visualisation of the aortic valve opening in all patients, and attempts to measure aortic valve area by planimetry can reasonably be made.

The results of the first published series by Hoffmann et $a l^{12}$ were very encouraging and supported our initial results, obtained with online aortic valve area measurements, which were quite satisfactory. ${ }^{22}$ These investigators obtained a correlation of $r=0.95$, with $P$ unspecified, between valve area determined by multiplane TOE and valve area determined by catheterisation, both of which were performed in all their patients. In the series of Tribouilloy et $a l_{,}{ }^{13}$ the results of TOE planimetry were very satisfactory as well, with $r=0.90, \mathrm{P}<0.0001$ between multiplane TOE planimetry and catheterisation, and $r=0.96, \quad \mathrm{P}<0.0001$ between multiplane TOE planimetry and the continuity equation. But in this study, catheterisation was performed in 25 patients only and the results of aortic valve area planimetry were compared with those of catheterisation in 25 patients and with those of continuity equation in 49 . In our study, we chose to compare the results of TOE planimetry to those of catheterisation and to those of the continuity equation, that is to the two methods accepted as criterion standards. Even if it is an admitted fact that the Gorlin formula suffers from limitations in the estimation of valve areas, especially in low flow states, ${ }^{23} 24$ aortic valve area determined by catheterisation remains the criterion standard against which new non-invasive methods must be judged. Furthermore, comparison with the continuity equation alone may alter the correlation.

In our study, as well as in Hoffmann's and Tribouilloy's, cardiac output was calculated using thermodilution. The Fick method would have been more appropriate, as thermodilution can be faulted in many situations, such as tricuspid regurgitation ${ }^{25}$ and low cardiac out- put, ${ }^{26}$ whereas aortic and mitral regurgitation seem to have no adverse influence on measurements. ${ }^{27} 28$

Our results were very different from those reported by these investigators. It should be noted that, to date, only two teams have reported good results with this method. Conversely, in a recent study from Cormier et $a_{1,29}$ the correlation between aortic valve area measured at TOE and at catheterisation was satisfactory only in patients with a mildly or moderately calcified aortic valve $(r=0 \cdot 80$, $P=0.005$ ) but was very poor in patients with heavily calcified valves $(r=0.13, P=0.69)$. This may go a long way towards explaining our bad results, since in our series most patients had severely calcified valves.

In our experience, there is a lack of agreement between the results of TOE planimetry and those of catheterisation, as well as between TOE planimetry and the continuity equation. TOE planimetry overestimates aortic valve area compared to the other two methods. The discrepancies between this method and the two established ones is unacceptable in clinical practice, as the need for surgery is decided on a very narrow range of aortic valve area values. Conversely, the continuity equation underestimates aortic valve area in comparison with catheterisation, but with a less important error than that generated by TOE planimetry. The discrepancies between several methods of estimation of aortic valve area have been reported previously ${ }^{8}$ and must be taken into account when considering the surgical indication in a precise case.

In fact, these poor results are not really surprising. On the one hand, the continuity equation and the Gorlin formula determine effective areas whereas planimetry measures anatomical area, which is expected to be greater than effective area. On the other hand, even if imaging quality is excellent with $5 \mathrm{MHz}$ TOE transducers, the problems that were common with transthoracic planimetry of calcific stenosed mitral valves are also encountered with aortic stenosis. Where there are heavy calcifications, obtaining a reliable drawing of aortic valve opening presents a challenge. In some cases, it was even impossible, and the feasibility of the method did not exceed $92 \%$ (48/52), $v 98 \%(51 / 52)$ for the continuity equation. Furthermore, the aortic valve is neither a planar nor a fixed orifice. As it is not planar, even if the best view for aortic valve visualisation is thoroughly searched, one cusp may be cut on a non-horizontal plane, what is a source of error in valve area planimetry. On the other hand, whatever the method used, orifice area calculation is based on the concept of a fixed orifice valve. In fact, it is now well established that effective aortic valve area varies during ejection in aortic stenosis, ${ }^{30}$ especially with regard to the transvalvar flow. ${ }^{31}$ During TOE examination, cardiac frequency usually increases, which may be a cause of increased transaortic flow and subsequently of increased aortic valve area.

Thus, if TOE is not effective for aortic valve area planimetry, is it useful for accurate mea- 
surement of left ventricular outflow tract diameter, in order to optimise continuity equation results? In our experience, as in that of Harpaz et $a^{3},^{32}$ the left ventricular outflow tract diameters measured by transthoracic and transoesophageal approaches correlated well, with an identical diameter or not more than a $2 \mathrm{~mm}$ difference between the two results in all 22 patients. Consequently, the results of continuity equation obtained with both diameters showed good agreement.

\section{CONCLUSIONS}

Despite previously reported satisfactory results, our study suggests that aortic valve area planimetry uing multiplane TOB is a diffeult, unreliable, and poorly reproducible method in the asseasment of aortic stenosis. This semilnvasive technique shows no superiority over the continuity equation, which remains the most effective non-invasive method for aortic valve area estimation.

We are indebted to Nancy Richardson and to Sophie Boucher for reviewing the manuscript.

1 Hatle L, Angelsen BA, Tromsdal A. Non-invasive assessment of aortic valve stenosis by Doppler ultrasound. $\mathrm{Br}$ ment of aortic valve sten
Heart $\subsetneq 1980 ; 43: 284-92$.

2 Hegraens L, Hatle L. Aortic stenosis in adults. Non-invasive estimation of pressure differences by continuous wave Doppler echocardiography. Br Heart I 1985;54: 396-404.

3 Currie PJ, Seward JB, Reeder OS, Vliettra RB, Brennahan DR, Bresnahan J. et al. Continuous=wave Doppler echocardiographic assessment of severify of calciflc aortic stenosis: a simultaneous Dopplet-catheter corfelative study in 100 adult patients. Circulation 1985;71:1162-9.

4 Teirstein P, Yeager M, Yock PG, Popp RL. Doppler echocardiographic measurement of aortic valve area in aortic stenosis: a noninvasive application of the Gorlin formula. $¥ \mathrm{Am}$ Coll Cardiol 1986;8:1059-65.

5 Oh JK, Taliercio CP, Holmes DJ, Reeder GS, Bailey KR, Seward JB, et al. Prediction of the severity of aortic stenosis by Doppler aortic valve area determination: prospective Doppler-catheterization correlation in 100 patients. $\mathcal{f}$ tive Doppler-catheterization correl.

6 Skjaerpe T, Hegrenaes L, Hatle L. Noninvasive estimation of valve area in patients with aortic stenosis by Doppler ultrasound and two-dimensional echocardiography. Circulation 1985;72:810-18.

7 Zoghbi WA, Farmer KL, Soto JG, Nelson JG, Quinones MA. Accurate noninvasive quantification of stenotic aortic valve area by Doppler echocardiography. Circulation 1986;73:452-9.

8 Fischer $\mathrm{JL}$, Haberer $T$, Dickson $D$, Henselmann $L$. Comparison of Doppler echocardiographic methods with heart catheterization in assessing aortic valve area in 100 patients with aortic stenosis. Br Hear $\mathcal{~}$ 1995;73:293-8.

9 Gorlin R, Gorlin SG. Hydraulic formula for calculation of the area of the stenotic mitral valve, other cardiac valves and central circulatory shunts. Am Heart $\mathcal{f} 1951$; 41:1-29.

10 Hofmann T, Kasper W, Meinertz T, Spillner G, Schlosser $V$, Just $H$. Determination of aortic valve orifice area in aortic valve stenosis by two-dimensional transesophageal aortic valve stenosis by two-dimensional transes

11 Stoddard MF, Arce J, Liddell NE, Peters G, Dillon S, Kupersmith J. Two-dimensional transesophageal Kupersmith J. Two-dimensional transesophageal echocardiographic determination of aortic valve area in adults with

12 Hoffmann R, Flachskampf FA, Hanrath P. Planimetry of orifice area in artic stenosis using multiplane transesophageal echocardiography. I Am Coll Cardiol 1993;22: 529-34.

13 Tribouilloy C, Shen WF, Peltier M, Mirode A, Rey J, Lesbre JP. Quantitation of aortic valve area in aortic stenosis with multiplane transesophageal echocardiography: comparison with monoplane

14 Sellers RD, Levy MJ, Amplatz K, Lillehei CW. Retrograde cardioangiography in acquired cardiac disease: technique, indications and interpretation of 700 cases. $A m \mathcal{F}$ Candiol 1964;14:437-47.

15 Fan PH, Kapur KK, Nanda NC. Color-guided Doppler echocardiographic assessment of aortic valve stenosis. F Am Coll Cardiol 1988;12:441-9.

16 Bland JM, Altman DG. Statistical methods for assessing agreement between two methods of clinical measurement. Lancit 1986;1:307-10

17 Britah Standards Inadtution. Precialon of teat methods I: Guide for the determination and reproducibility for a otandard teut method (BS 5497, part 1), London: BSI, 1979.

18 Panidis IP, Mints GS, Ross $J_{1}$ Value and Imitations of Doppler utrasound in the evaluation of eartie otenosis. A Dopplez ultrasound in the evaluaton of aortie stenosis. A $1996113: 150=8$

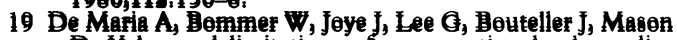
D. Value and limitations of eress=8ectional echocardio= graphy of the aortic valve in the diagnosis and quantification of valvular aortic stenosis. Circulation 1980;62: 304-12.

20 Veyrat C, Gourtchiglouian C, Dumora P, Abitbol G, Sainte Beuve D, Kalmanson D. A new non-invasive estimation of the stenotic aortic valve by pulsed Doppler mapping. Br Heart $\mathcal{F}$ 1987;57:44-50.

21 Veyrat C, Legeais S, Gourtchiglouian C, Sainte-Beuve D, Abitbol G, Kalmanson D. Nouvelle approche méthodologique quantitative des jets valvulaires gauches en imagerie Doppler couleur. Arch Mal Coeur 1989;82: en imagerie.

22 Bernard YF, Meneveau NF, Anguenot TJ, Zhang J, Schiele FJ, Vuillemenot AR, et al. Accuracy of aortic valve area measurement by multiplane transesophageal echocardiography in aortic utenosis [abstract]. I Am Coll Cardiol 1995;25 muppl 1:309A

23 Cannon SR, Rehards Ki, Crawford M. Hydraulic estimation of stenotic orfice area: a correction of the Gorlin formula. Cinculation 1985;71:1170-8.

24 Segal f, Lefner Df, Miller DC, Mitchell RS, Alderman BA, Popp RL. When should Doppler-determined valve area be better than the Gorlin formula? Variation in hydraulic constants in low flow states. $\mathcal{F} \mathrm{Am}$ Coll Cardiol 1987;9: 1294-305.

25 Lipkin DP, Poole-Wilson PA. Measurement of cardiac output during exercise by the thermodilution and direct Fick techniques in patients with chronic congestive heart failure. Am $f$ Cardiol 1985;56:321-4.

26 Van Grondelle A, Ditchey RV, Groves BM, Wagner WW, Reeves JT. Thermodilution method overestimates low Reeves JT. Thermodilution method overestimates low
cardiac output in humans. Am $f$ Physiol 1983;245: H690-2.

27 Hillis LD, Firth BG, Winniford MD. Analysis of factors affecting the variability of Fick versus indicator dilution measurements of cardiac output. Am $\mathcal{F}$ Cardiol 1985;56: 764-8.

28 Hamilton MA, Stevenson LW, Woo M, Child JS, Tillisch $\mathrm{JH}$. Effect of tricuspid regurgitation on the reliability of the thermodilution cardiac output technique in congestive heart failure. Am $\mathcal{F}$ Cardiol 1989;64:945-8.

29 Cormier B, Iung B, Porte JM, Barbant S, Vahanian A. Value of multiplane transesophageal echocardiography in determining aortic valve area in aortic stenosis. Am $\mathcal{f}$ Cardiol 1996;77:882-6.

30 Badano L, Cassottana P, Bertoli D, Carratino L, Lucatti A, Spirito $P$. Changes in effective aortic valve area during ejection in adults with aortic stenosis. Am $f$ Cardiol 1996;78: 1023-8.

31 Paulus WI, Sys SU, Heyndrickx GR, Andries E. Orifice variability of the stenotic aortic valve: evaluation before and after balloon aortic valvuloplasty. $f \mathrm{Am}$ Coll Cardiol 1991;17:1263-9.

32 Harpaz D, Shah P, Bezante G, Heo M, Stewart S, Hicks GL, et al. Transthoracic and transesophageal echocardiographic sizing of the aortic annulus to determine prosthesis size. Am $\mathcal{F}$ Cardiol 1993;72:1411-17. 